Rev. Biol. Trop., 47(4): 729-737, 1999

www.ucr.ac.cr www.ots.ac.cr www.ots.duke.edu

\title{
Aporte de hojarasca y renovación foliar del manglar en un sistema estuarino del Sureste de México
}

\author{
Ma. Teresa Barreiro-Güemes
}

Departamento El Hombre y su Ambiente, Universidad Autónoma Metropolitana. Unidad Xochimilco. Calzada del Hueso 1100. Col. Villa Quietud, Coyoacán México D.F. 04960

Fax: (52) (5) 723-59-64 Correo electrónico: bteresa@ cueyatl.uam.mx

Recibido 20-VIII-1998. Corregido 9-VI-1999. Aceptado 9-VI-1999.

\begin{abstract}
The patterns of mangrove litter production were studied in Pom-Atasta, a estuarine system linked to Terminos Lagoon, Campeche coast, Mexico. Four sampling sites with contrasting conditions of tidal influence were selected. Ten litter baskets placed randomly in each site for litter fall evaluation, were emptied monthly during an annual cycle (1995-1996). Litter material was classified, dried and weighed. The flowering season for Avicennia germinans was from June through September; Rhizophora mangle had flowers and hypocotyls year round. The most productive site was riverine forest dominated by $A$. germinans in the area with least tidal influence and hypohaline conditions, low annual salinity variation and lime-clay soils. Average litter production varied between 1.8 and $4.5 \mathrm{~g} \mathrm{~m}-2$ day-1 and was closely related to regional climatic periods. In October 1995, two hurricans: Roxane and Opal, blew in the region and caused an extraordinary defoliation with litter production between 8 a $12 \mathrm{~g} \mathrm{~m}$-2day- 1 These figures greatly exceed the amounts registered for mangroves in the Gulf of Mexico, the Caribbean and the Equator. The leaf renewal rate was 129-392 days.
\end{abstract}

Key words: Mangroves, leaf litter production, leaf renewal time, Pom-Atasta, Campeche coast.

Los manglares de México de ambos litorales han sido estudiados por numerosos autores: Flores-Verdugo et al. (1992) y Tovilla (1994) han publicado revisiones sobre este tema. Para el sistema estuarino Pom-Atasta, asociado a la laguna de Términos en el estado de Campeche, no se han publicado estudios sobre la comunidad de manglar. En este sistema el manglar alcanza un gran desarrollo; en visitas previas a la zona, se han observado individuos de tallas mayores que las registradas en otros lugares del Golfo de México y del Caribe (obs.pers) Este sistema está asociado a la laguna de Términos y ha sido identificado como importante subsidiario de materia orgánica a la propia laguna y a la Sonda de Campeche (Ayala et al. 1993). Esta región está siendo afectada por la extracción petrolera y por la creación de infraestructura relacionada con ésta. Por otra parte es una fuente importante de productos pesqueros y tiene potencial para la acuicultura (Aguirre $e t$ al.1998). El estudio de la producción primaria de los manglares de esta región, complementa el marco ecológico de referencia para basar las acciones de ordenamiento ecológico que se requieren para esta zona.

El sistema estuarino Pom-Atasta, en la porción occidental de la Laguna de Términos, Campeche (Fig. 1), cubre $190 \mathrm{~km}^{2}$ con una profundidad promedio de $2.7 \mathrm{~m}$. Está comprendido dentro de la llanura costera formada por los ríos Grijalva y Usumacinta (GutiérrezEstrada et al. 1982). 


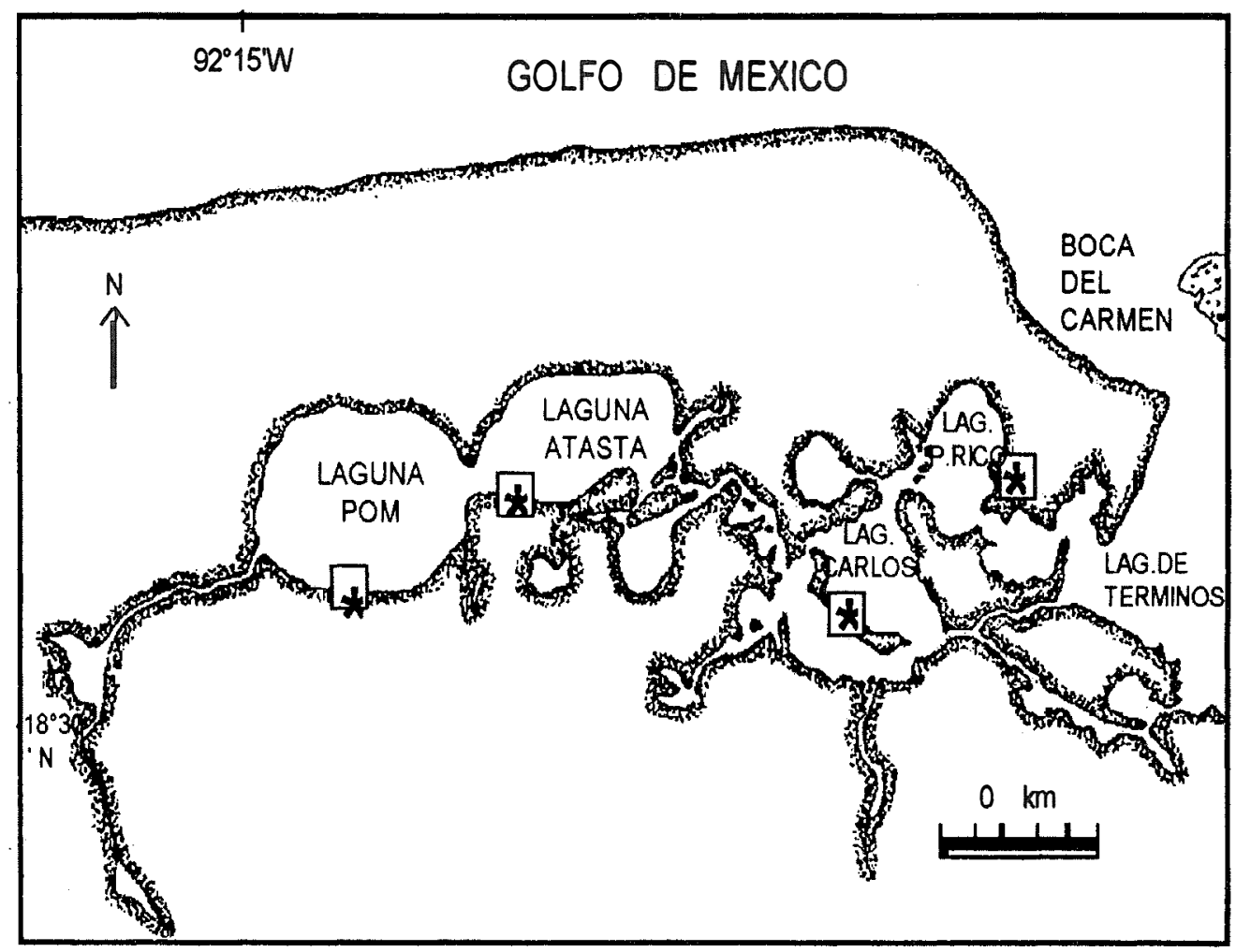

Fig. 1. Sistema estuarino Pom-Atasta, Campeche. Sitios de muestreo del manglar. $\left({ }^{*}\right)$

Fig. 1. Pom-Atasta system, Campeche. Mangrove sampling sites $\left({ }^{*}\right)$

Presenta un gradiente estuarino desde las porciones más altas, hasta la desembocadura, con un intervalo de salinidad de 2 a 26 ups. que varía dependiendo de la época climática del año, la precipitación pluvial y la influencia de marea (Trejo 1994, Bernal-Becerra 1995). El clima es tropicalhúmedo con precipitación de 1100 a $2000 \mathrm{~mm}$, así se establecen las temporadas de lluvias (de junio a octubre), de secas (de febrero a mayo) y en cuanto al viento, de nortes (de noviembre a enero).

\section{MATERIALES Y MÉTODOS}

Se eligieron cuatro rodales sujetos a condiciones contrastantes en cuanto a su régimen hidrológico: dos en la zona de mayor influencia mareal: la Laguna de Puerto Rico, y la Laguna de Carlos, y dos en la zona de menor influencia mareal con régimen limnético: la Laguna de Atasta y la Laguna del Pom (Fig. 1)
El rodal de la Laguna de Puerto Rico tiene las características de un manglar de borde sujeto a fuertes fluctuaciones de salinidad a lo largo del año. Es un bosque joven, mixto de Avicennia germinans L. y Laguncularia racemosa Gaert, de alta densidad. El rodal del islote de la Laguna de Carlos corresponde a un bosque sobrelavado, formado por la colonización de bancos poco profundos, consolidados por praderas de Halodule wrightii Ascherson, con Rhizophora mangle L. como especie colonizadora y A. germinans como dominante. El rodal de Atasta es de bosque ribereño en un área interna del sistema, con poco relieve, donde la renovación de aguas ocurre muy lentamente. Está dominado por A. germinans, con individuos de gran porte aunque con baja densidad. El rodal de la Laguna de Pom, es un bosque ribereño con suelos arcillosos, drenaje lento, condiciones de salinidad poco variables a lo largo del año e individuos de gran talla, dominado por R.mangle (Barreiro en prep.) (Cuadro 1) 


\section{CUADRO 1}

Características estructurales de los manglares estudiados

TABLE 1

Structural characteristics of mangroves at sampling sites

$\begin{array}{lccccc}\text { Zona } & \text { Tipo fisiográfico } & \begin{array}{c}\text { Diámetro promedio } \\ (\mathrm{cm})\end{array} & \begin{array}{c}\text { Area basal } \\ \left(\mathrm{m}^{2} \mathrm{ha}^{-1}\right)\end{array} & \begin{array}{c}\text { Densidad } \\ \left(\text { ind.ha }{ }^{-1}\right)\end{array} & \begin{array}{c}\text { Altura promedio } \\ (\mathrm{m})\end{array} \\ \text { Puerto Rico } & \text { Borde } & 10.9 & 32.15 & 3100 & 7.4 \\ \text { Carlos } & \text { Sobrelavado } & 10.3 & 31.81 & 5000 & 4.6 \\ \text { Atasta } & \text { Ribereño } & 23.5 & 79.3 & 1650 & 12.5 \\ \text { Pom } & \text { Ribereño } & 19 & 67.34 & 2200 & 11.3\end{array}$

Tomado de Barreiro (en prep.)

El aporte de hojarasca de cada rodal se evaluó, en términos de materia orgánica (peso seco), aportada por tiempo y por superficie. Para esto se colocaron aleatoriamente en cada rodal seleccionado diez trampas de forma cuadrangular de $0.25 \mathrm{~m}^{2}$. de superficie, construidas con tubo de polivinilo, con una bolsa de malla plástica de $1 \times 1 \mathrm{~mm}$ de luz, suspendidas entre el follaje para recolectar el material desprendido por los árboles, en el área interceptada (Brown 1985). El método de recolecta de hojarasca en trampas o cestas de diversas formas ha sido utilizado con éxito por Snedaker y Brown (1981) y Pool et al. (1975) en manglares de Florida y el Caribe. Este método es sencillo, aunque requiere de cuidados en la selección de un material resistente a la humedad para la construcción del marco y de una malla resistente, que sea suficientemente fina para retener el material y a la vez permitir su aireación. Además debe tenerse cuidado en la colocación de las trampas, para evitar que sean derrumbadas o volteadas por el viento.

Las trampas se colocaron en marzo de 1995 en los cuatro sitios seleccionados. Las trampas se revisaron a intervalos mensuales de abril de 1995 a junio de 1996, recolectándose el material, el cual fue seleccionado por especie y por tipo de material (hojas, estípulas, cortezas, ramas, flores, semillas e hipocotilos) y luego fue secado a $70^{\circ} \mathrm{C}$ hasta obtener el peso seco constante. La tasa de renovación de follaje para las principales especies se calculó como una relación de la biomasa defoliada (exclusivamente hojas) sobre la biomasa foliar en pie; este valor se estimó con base en las ecuaciones alométricas desarrolladas por Barreiro (en preparación). Estas fueron calculadas defoliando totalmente 15 individuos de diferentes tallas y graficando el peso seco del follaje contra el valor del cuadrado del diámetro por la altura. Con estos valores se estimaron la ordenada al origen y al pendiente de estas ecuaciones, con coeficientes de correlación (r2) de 0.91 para $R$. mangle y 0.92 para A.germinans

Para R. mangle: $\mathrm{Y}=(60.7) \mathrm{X}^{0.5}$

Para A.germinans $\mathrm{Y}=(54.3) \mathrm{X}^{0.48}$

Donde: $Y=$ biomasa foliar seca en gramos, $X=(\text { dap })^{2} h$

dap $=$ diámetro a la altura del pecho en $\mathrm{cm} ; \mathrm{h}=$ altura en $\mathrm{m}$

Los registros de salinidad de las zonas muestreadas se tomaron del banco de datos hidrológicos existente para la zona en el Laboratorio de Ecología Costera del Departamento el Hombre y su Ambiente. Los datos climáticos se tomaron de la estación meteorológica de Ciudad del Carmen, Campeche.

Se calcularon las medidas de estadística descriptiva de cada uno de los sitios muestreados: Media, moda, rango y desviación estándar. Se realizó un análisis de varianza, ANOVA, para estimar diferencias significativas entre zonas y entre periodos climáticos (Daniel 1983). 


\section{CUADRO 3}

Composición de la hojarasca en las distintas zonas. Promedio de registros mensuales. (g.m-2dia-1, peso seco)

TABLE 3

Litter composition at sampling sites, monthly average (g.m-2day-I dry weight)

Laguna de Puerto Rico

Especie

R. mangle

A.germinans.

L.racemosa

Misceláneo*

Total

Laguna de Carlos

R. mangle

A.germinans.

L.racemosa

Misceláneo*

Total

Laguna de Atasta

$R$. mangle

A.germinans.

L.racemosa

Misceláneo*

Total

Laguna de Pom

$R$. mangle

A.germinans.

L.racemosa

Misceláneo*

Total

Hojas y
estípulas

0.14

1.30

0.15

\subsection{4}

1.22

0.18

\subsection{8}

2.10

0.36

\subsection{4}

0.66

0.22
0.20

0.02

0.03

0.12

0.05

Flores

0.03

-

$-$

0.34
Semillas o

hipocotilos

$\begin{array}{ccc}- & 0.14 & 7.7 \\ - & 1.33 & 73.5 \\ - & 0.15 & 8.3 \\ & 0.19 & 10.5 \\ & 1.81 & 100 \\ - & & \\ - & 0.96 & 33.3 \\ - & 1.27 & 44.1 \\ & 0.18 & 6.2 \\ & 0.47 & 16.4 \\ 0.08 & 2.88 & 100 \\ - & & \\ & 0.46 & 15 \\ & 2.12 & 68.8 \\ & 0.39 & 12.7 \\ 0.53 & 0.11 & 3.5 \\ 0.06 & 3.08 & 100 \\ - & & \\ & 2.91 & 64.6 \\ & 1.26 & 27.9 \\ & 0.22 & 4.8 \\ & 0.12 & 2.7 \\ & 4.51 & 100\end{array}$

*Incluye: ramas, cortezas y material no identificado

que tiene un régimen de salinidad ligeramente menor (de 1.9 a 21 ups, con una media de 11 ups.) que la Laguna de Puerto Rico (de 3 a 24 ups, con una media de 14 ups.). La composición del follaje en estas dos zonas está dominada por A. germinans seguida por $R$. mangle; la especie $L$. racemosa aporta muy poco follaje, sin embargo esta especie está presente de manera abundante en la laguna de Puerto Rico. Su escasa defoliación puede deberse a que se trata de árboles jóvenes de pequeño porte. La producción y la composición de la hojarasca por especies en las distintas zonas se expresan en el Cuadro 3.

Patrón estacional de producción de flores y semillas: Las flores, frutos y semillas estuvieron presentes en la composición de la hojarasca, su presencia indica las temporadas de floración de las especies en las distintas zonas. Las flores de R.mangle estuvieron presentes casi.todo el año en Atasta y Pom, con excepción de la época de nortes, que corresponde al otoño-invierno. Los hipocotilos de esta especie se presentaron durante la época de lluvias (junio a septiembre) (Cuadro 4).

Las flores y semillas de A.germinans solo aparecieron de junio a septiembre y las de $L$ racemosa solo se presentaron en Atasta durante el verano.

Tasa de renovación de follaje: La tasa de renovación de follaje para $A$. germinans y $R$.mangle, para cada una de las zonas se detalla en el Cuadro 5. La tasa de renovación estimada oscila entre 129 y 397 días, y en general es más alta en los sitios de mayor influencia de marea que en los bosques situados en la parte interna del sistema, los cuales, según Barreiro 


\section{CUADRO 4}

Presencia de flores, semillas e hipocotilos durante el ciclo anual (g. $\mathrm{m}^{-2}$ dia $^{-1}$, peso seco)

\section{TABLE 4}

Flowers, seeds and hipocotyls during the annual cycle (g.m $m^{-2}$ day-1, dry weight)

Esp. Jul. Ag. Sep. Oct. Nov. Dic. Ene. Feb. Mar. Abr. My. Jun.

Laguna de Puerto Rico

R.mangle

A. germ.

$0.13 \quad 0.11$

0.12

Laguna de Carlos

R.mangle

$\begin{array}{lll}1.10 & 0.12 & 0.14\end{array}$

A. germ.

0.6

\section{Laguna de Atasta}

\begin{tabular}{|c|c|c|c|c|c|c|c|c|c|c|}
\hline R.mangle & & 1.19 & 0.63 & 0.2 & & 0.14 & 0.1 & 0.6 & 0.3 & 0.2 \\
\hline A. germ. & & 0.24 & & & & & & & & \\
\hline L. rac. & & 0.36 & & & & & & & & \\
\hline Laguna de Pom & & & & & & & & & & \\
\hline R.mangle & 1.41 & 1.18 & 1.32 & & 0.22 & 1.31 & 1.29 & 0.7 & 1.6 & 1.39 \\
\hline A. germ. & 0.18 & 0.41 & & 0.13 & & & & & & \\
\hline
\end{tabular}

\section{CUADRO 5}

Tasa estimada de renovación de follaje en las zonas estudiadas

TABLE 5

Estimated foliar renewal rate in several sites

Zona

Puerto Rico

Atasta

Pom
Especie

A. germinans
R. mangle
Total

A. germinans

R. mangle

Total
A. germinans
R. mangle
Total
A. germinans
R. mangle
Total

Biomasa foliar en pié

(kg ha ${ }^{-1}$ peso seco)*

2173

298

2471

2590

1088

3678

6249

679

6928

1144

7974

9118
Hojarasca

$\left(\mathrm{kg} \mathrm{ha}^{-1} \mathrm{año}^{-1}\right.$ )

(solo hojas)

4781

511

5292

4453

3066

7519

7300

693

7993

2409

7480

9989
Tasa de renovación (días)

165

213

170

212

129

178

312

357

317

173

392

334

$\left.{ }^{*}\right)$ Los datos de biomasa foliar en pié fueron tomados de Barreiro, en preparación. 


\section{CUADRO 6}

Probabilidad estimada por un análisis de varianza, de diferencias significativas en la producción de hojarasca entre zonas y entre temporadas

\section{TABLE 6}

Estimated probability of significant differences in litter fall between sites and between seasons

$\begin{array}{lcccc}\text { Zonas } & \text { Puerto Rico } & \text { Carlos } & \text { Atasta } & \text { Pom } \\ \text { Puerto Rico } & - & 0.006 & 0.0001 & 0.0002 \\ \text { Carlos } & - & - & 0.04 & 0.001 \\ \text { Atasta } & - & - & - & 0.01 \\ \text { Pom } & - & - & - & - \\ & & & & \\ \text { Temporadas } & \text { Lluvias } & \text { Nortes } & \text { Seca } \\ \text { Lluvias } & - & 0.29 & 0.02 \\ \text { Nortes } & - & & 0.03 \\ \text { Seca } & - & - & -\end{array}$

$\mathrm{P}<0.05$ Se consideró significativa

(en preparación) son de mayor porte y mayor densidad. La tasa de renovación foliar es más lenta en estos los bosques que por otra parte, presentaron un mayor aporte de hojarasca.

Validación estadística: Los resultados del ANOVA para la comparación entre las cuatro zonas estudiadas y las tres temporadas climáticas, se expresan en el Cuadro 6, en términos de probabilidad de que las diferencias encontradas sean debidas al azar. Se consideró que una probabilidad menor a 0.05 representa- ba diferencias significativas entre los universos muestreados. Como se observa en el Cuadro 4, hay una diferencia altamente significativa entre la zona de Pom y la de Puerto Rico, o sea entre las condiciones más extremas del Sistema. La menor diferencia se encontró entre la Laguna de Carlos y la de Atasta, que representan los puntos medios del gradiente de condiciones de salinidad, que van desde la condición mesohalina hasta la limnética.

En cuanto a las temporadas la diferencia más significativa en la producción de hojarasca fue entre la temporada de lluvias y la de sequía.

\section{DISCUSIÓN}

La producción primaria de los bosques de manglar se relaciona con la estructura fisiográfica del bosque (Lugo y Snedaker 1974) así como con el régimen hídrico al que esté sujeto, la influencia de marea, los vientos y en general las características climáticas y edáficas (Uncles et al. 1992, Rivera-Monroy et al. 1995).

En los cuatro sitios contrastados se mostraron diferencias significativas en la producción de hojarasca, ya que corresponden a tipos fisiográficos diferentes y están sujetos a diferentes regímenes de salinidad. (Cuadro 7) (Barreiro en preparación):

El rodal en la Laguna de Puerto Rico, que es un manglar de borde sujeto a fuertes fluctuaciones de salinidad a lo largo del año presentó

\section{CUADRO 7}

Producción total de hojarasca por sitios y tipos fisiográficos

TABLE 7

Total litter production in mangrove sites and physiographic types

\begin{tabular}{|c|c|c|c|c|c|c|c|c|}
\hline \multirow[t]{2}{*}{ Zona } & \multirow{2}{*}{$\begin{array}{c}\text { Tipo } \\
\text { fisiográfico }\end{array}$} & \multicolumn{3}{|c|}{ Salinidad ups. } & \multirow{2}{*}{$\begin{array}{c}\text { Area basal } \\
\left(\mathrm{m}^{2} \mathrm{ha}^{-1}\right)\end{array}$} & \multirow{2}{*}{$\begin{array}{l}\text { Densidad } \\
\left(\text { ind.ha }^{-1} \text { ) }\right.\end{array}$} & \multirow{2}{*}{$\begin{array}{c}\text { Altura } \\
\text { promedio. (m) }\end{array}$} & \multirow{2}{*}{$\begin{array}{l}\text { Producción } \\
\text { hojarasca } \\
\text {.kgha-1año-1 }\end{array}$} \\
\hline & & Min. & Mex & Media & & & & \\
\hline Puerto Rico & Borde & 3 & 24 & 14 & 32.15 & 3100 & 7.4 & 6606 \\
\hline Carlos & Sobrelavado & 2 & 21 & 11 & 31.81 & 5000 & 4.6 & 10512 \\
\hline Atasta & Ribereño & 2 & 12 & 7 & 79.3 & 1650 & 12.5 & 11242 \\
\hline Pom & Ribereño & 0 & 10 & 3 & 67.34 & 2200 & 11.3 & 16461 \\
\hline
\end{tabular}

- Datos estructurales tomados de Barreiro (en prep.) 
la menor producción de hojarasca; por su ubicación, esta zona fue la menos influenciada por el efecto de los ciclones que se presentaron en el mes de octubre. El rodal del islote de la Laguna de Carlos, corresponde a un bosque sobrelavado; fue fuertemente influenciado por los ciclones, lo que hizo su producción muy fluctuante al menos por este ciclo anual. El rodal de Atasta corresponde a un bosque ribereño, al igual que el de la Laguna de Pom, estos presentan condiciones de salinidad poco variables a lo largo del año con individuos de gran talla y presentaron la mayor producción de hojarasca.

El patrón estacional de producción de hojarasca fue similar en las cuatro zonas y responde a las variaciones de los periodos climáticos que se definen en la zona. Durante la época de lluvias y la de nortes se aporta el mayor volumen de hojarasca a la laguna, lo cual fertiliza las aguas favoreciendo aumentos en la biomasa de consumidores. Bernal-Becerra (1995) en un estudio de la dinámica de la comunidad ictiológica, concluye que es en esa época en la que se presenta la mayor biomasa de peces.

En el ciclo anual estudiado (1995-96) se presento un evento meteorológico importante: la presencia de los ciclones Roxane y Opal que azotaron la región. Este evento causó una extraordinaria defoliación en tres de las zonas estudiadas con aportes de hojarasca de 8 a 12 $\mathrm{g} \mathrm{m}^{-2}$ promedio anual diario, cifras que exceden en mucho a las registradas para manglares de México, del Caribe y del Ecuador, que son del orden de 1 a $4 \mathrm{~g} \mathrm{~m}^{-2}$ (Cintrón y Shaeffer-Novelli 1992). Sin embargo, esta zona, aun sin considerar el efecto de los ciclones tiene una producción mayor que otros bosques en la Laguna de Términos: El bosque de Estero Pargo produce de 4040 a $7930 \mathrm{~kg} \mathrm{ha}^{-1}$ $\mathrm{año}^{-1}$ (Day et al. 1996) y el bosque de Boca Chica, aporta entre 7700 y $12060 \mathrm{~kg} \mathrm{ha}^{-1}$ año-1 (Day et al. 1987). Los ciclones causan una fertilización extraordinaria de las aguas de los sistemas estuarino lagunares, que seguramente redundan en aumentos en la producción secundaria de estos sistemas; sin embargo también pueden provocar eutroficación temporal con abatimiento de oxígeno disuelto y mortalidad de peces. La presencia de situaciones extraordinarias en un ciclo de estudio, lleva a la reflexión de la necesidad de hacer registros sistemáticos en periodos largos de tiempo y dar seguimiento a la producción de hojarasca de los manglares.

La tasa de renovación de follaje al parecer se relaciona de manera inversa con el grado de desarrollo del bosque, ya que la tasa de renovación estimada para los bosques de pequeño porte fue casi del doble que la de los rodales donde dominaron árboles grandes.

Las diferencias significativas en la producción de hojarasca entre temporadas climáticas y entre zonas con condiciones distintas en cuanto a régimen hídrico, tipo de sedimento y fisionomía hacen evidente la respuesta de las comunidades de manglar a las condiciones más o menos favorables del hábitat.

\section{AGRADECIMIENTOS}

Agradezco a Alfonso Vázquez Botello, Francisco Flores Verdugo y José Luis Arredondo Figueroa, la revisión y sugerencias al manuscrito, y la colaboración de Francisco Vera Herrera, Hernán Guillen y Andrés Reda, de la Estación Carmen del Instituto de Ciencias del Mar y Limnología de la Universidad Nacional Autónoma de México, su apoyo en campo; a Ma. Teresa López Barreiro y a Jaime Vargas su participación en el procesamiento de muestras y datos.

\section{RESUMEN}

Los patrones de producción de hojarasca fueron estudiados en Pom-Atasta, un sistema estuarino asociado a la Laguna de Términos Campeche, México. Se seleccionaron cuatro rodales de manglar, contrastantes por sus condiciones de influencia de marea. Se colocaron al azar diez trampas en cada sitio para la colecta de hojarasca, estas fueron revisadas a intervalos mensuales durante un ciclo anual (1995-96). El material recolectado fue clasificado, secado y pesado. La floración de Avicennia germinans se presentó desde junio hasta septiembre; $R h i$ zophora mangle presentó flores e hipocotilos todo el 
año. Los bosques más productivos fueron los ribereños dominados por $A$. germinans en la zona de menor influencia de marea, con condiciones hipohalinas de escasa variación anual y substratos limo-arcillosos. La producción promedio de hojarasca varió entre 1.8 y $4.5 \mathrm{~g} \mathrm{~m}^{-2}$ día ${ }^{-1}$ y respondió a los periodos climáticos de la región. En octubre de 1995 los ciclones Roxane y Opal se presentaron en la región y causaron una extraordinaria defoliación con aportes de hojarasca de 8 a $12 \mathrm{~g} \mathrm{~m}^{-2} \mathrm{dí}^{-1}$ cifras que exceden a las registradas para manglares de México, del Caribe y del Ecuador. La tasa de renovación de follaje fue de 129-392 días.

\section{REFERENCIAS}

Aguirre-León, A, A. Bernal-Becerra \& S. Díaz-Ruiz. 1998. Ecología de peces en sistemas fluvio-deltaícos: Estudio para el sistema Pom-Atasta Campeche. Serie Académicos: 28 Ciencias Biologicas y de la Salud. Universidad Autónoma Metropolitana-Xochimilco. México, D.F. 65 p.

Ayala, L.A, A. Aguirre- León, O. Avilés, M.T. Barreiro \& J.L. Rojas-Galaviz. 1993. Peces de sistemas fluviolagunares, Laguna de Términos Campeche. p 596608.. In S.Salazar-Vallejo \& N.E. González (eds). Biodiversidad Marina y Costera de México. Comisión Nacional para el Conocimiento y uso de la Biodiversidad- Centro de Investigación de Quintana -Roo. México D.F.

Bernal-Becerra, A. 1995. Dinámica estacional de la comunidad nectónica en el sistema fluvio-lagunar deltáico PomAtasta asociado a la Laguna de Términos Campeche, México. Informe de Servicio Social, Universidad Autónoma Metropolitana Unidad Xochimilco. México D.F.

Brown, M. 1985. Mangrove leaf litter production and dinamics, p 231-251 In. S. Snedaker y J. Snedaker (eds). The mangrove ecosystem research methods. United Nations Educational, Scientific \& Cultural Organization. Bungay, Reino Unido

Cintrón, G. \& Y. Shaeffer-Novelli. 1992. Ecology and management of New World mangroves, p 234-257 $m \mathrm{U}$. Seeliger (ed.). Coastal Plant Communities of Latin America, Academic, Londres.

Daniel, W.W. 1983. Bioestadística básica para el análisis de las ciencias de la salud. Limusa, México D.F. 485 p.

Day, J.W, W.H. Conner, F. Ley-Lou, R. Day \& N.A. Machado. 1987. The productivity and descomposition of mangrove forest in Laguna de Términos México. Aquat. Bot. 27: 267-284
Day, J.W, C. Coronado-Molina, F.R. Vera -Herrera, R.Twilley, V.H. Rivera-Monroy, H. Alvarez-Guillén, R. Day \& W. Conner. 1996. A 7 year record of above ground net primary production in a southeastern mexican mangrove forest. Aquat. Bot. 55: $39-60$

Flores-Verdugo, F, F. González Farías, D.S. Zamorano \& P. Ramírez García. 1992. Mangrove ecosystems of the Pacific Coast of México: Distribution, structure, litterfall and detritus dynamics, p. 269-287 In: U. Seeliger (ed.). Coastal Plant Communities of Latin America. Academic, Londres.

Gutiérrez-Estrada, M, V. M. Malpica \& J. Mar ínez. 1982. Geomorfología y sedimentos recientes de Sistema Lagunar Atasta-Pom Campeche México. Ann. del Inst. de Cienc. del Mar y Limn. Universidad Nacional Autónoma de México 9: 89-100.

Lugo, A. \& S. Snedaker. 1974. The Ecology of Mangroves. Ann. Rev. Ec. Sys. 5: 39-64

Pool, D.J, S.C. Snedaker, \& A.E. Lugo. 1975. Litter production in mangrove forest in South Florida and Puerto Rico, p 213-237 In: G. Walsh, S. Snedaker \& H. Teas. (eds). Proceedings of International Symposium on Biology and Management of Mangroves. Vol.1 Gainesville Institute of Food and Agriculture Sciences, University of Florida.

Rivera-Monroy, V, J.W.Day, R.R.Twilley, F. Vera-Herrera \& C.Coronado-Molina. 1995. Flux of nitrogen and sediment in a fringe mangrove forest in Términos Lagoon. México. Est. Coast. Shelf Sci. 40: 139-160.

Snedaker, S.C. \& M.S. Brown. 1981. Primary Productivity of Mangroves. p. 477-486. $\mathrm{In}$ : C.C. Black \& A. Mit sui (eds). CRC Handbook Series of Biosolar Resourses. Vol I: Basic Principles. CRC, West Palm Beach. Florida.

Tovilla, H.C. 1994. Los Manglares. p 371-423 In: G. De la Lanza \& C.M. Cáceres (eds.) Lagunas Costeras y el Litoral Mexicano Universidad Nacional Autónoma de México-Universidad Autónoma de Baja California Sur. La Paz Baja California Sur.

Trejo O. 1994. Análisis de la estructura de la comunidad nectónica del Sistema Pom-Atasta adyacente a la Laguna de Términos Informe de Servicio Social Universidad Autónoma Metropolitana-Xochimilco, México D.F. 24p.

Uncles, R.J, W.K. Wong \& J.E. Ong. 1992. Intratidal fluctuations in stratification within a mangrove estuary. Hydrobiology. 247:163-171. 